\title{
Curve Distance Measure for Quantitative Evaluation of Myocardial Boundary Tracking
}

\author{
Adhi Harmoko Saputro ${ }^{1}$, Mohd. Marzuki Mustafa ${ }^{2}$, Aini Hussain ${ }^{3}$ \\ Department of Electrical, Electronic and Systems Engineering \\ Universiti Kebangsaan Malaysia, Bangi 43600, Malaysia \\ Email: 'adhi@eng.ukm.my, ${ }^{2}$ marzuki@eng.ukm.my,3aini@eng.ukm.my
}

\begin{abstract}
Myocardial boundary tracking is part of feature extraction task for evaluating human cardiac abnormalities. In this case, the feature of myocardial contraction is extracted in the form of the boundary shape movement and alteration such as shape motion, shape deformation. In this paper, a curve distance measure is proposed for evaluating performance of myocardial boundary tracking techniques. The curve is constructed of $9-14$ control points along the myocardial boundary which is tracked to all frames in the cardiac cycle between the end diastole and the end systole. The curves distance measure implemented in this study is average of absolute shortest distance between estimated curve and ground truth curve. Both of curves are generated using b-spline interpolation technique. The estimated control point is the yield of the tracking process of the initial point in motion vector space. In this experiment, eight seven motion estimation techniques such as Black Anandan, Brox, Gautama, Gurner Farneback, Horn Shuck, LK, LK-Pyr and proposed method are compared to know their performance in tracking on echocardiography image. The database sets are generated to test the performance algorithm that comprising set sequences of ultrasound images with known initial and final boundary. The known vector motion is used to generate the ground truth of synthetic myocardial boundary tracking. The real clinical data that have initial and final boundary generated by cardiologist are also used to evaluate the performance. Accordingly, results obtained showed that quantitative measures can be obtained in term of curve distance mean of initial and final boundary which is useful for performance evaluation of the aforementioned techniques with the proposed method recorded superiority over other techniques. In short, the curve distance measure described in here is useful and provides means of evaluating performance of myocardial boundary tracking quantitatively.
\end{abstract}

Keywords - Boundary Tracking; Quantitative Evaluation; Optical Flow; Echocardiography

\section{INTRODUCTION}

Quantitative evaluation is an important term in evaluating a proposed novel technique then compared with other established technique. The improvement performance of a new technique is compared quantitatively by implementing the new and established technique to the simulated or real data experiment. There are some quantitative evaluations in image processing correlating with this work such angular error [1] or cumulative percentage error [2] to evaluate the motion vector result of optical flow technique, Hausdorff [3] or Euclidian distance to evaluate boundary detection technique.

The tracking of any object in echocardiography image sequence is current active research. It has a goal to find a technique to track the movement of non-rigid and noisy object accurately. The noisy of echocardiography image contribute significantly in the error result of the tracking computation. In [4], authors proposed a fusion information in the information space by resolving the uncertainties from the dynamic system. Mikic et al. have been used an active contours which guided by optical flow to track echocardiographic sequence [5]. In [6], authors combined the snake and optical flow technique to improve tracking performance in contract echocardiography. In previous work [7], we proposed a tracking method by point tracking along myocardial boundary in high accurate motion vector space.

Among the tracking algorithm evaluation, the mean absolute distance (MAD) [4], mean sum of squared distances (MSSD) [8] have been used to compute distance between the estimated point and the ground truth point. In this paper, we try to improve the deficiencies of both MAS and MSSD algorithm in evaluating myocardial boundary tracking the echocardiography cases. The goal of this paper is to present a new solution for the purpose of evaluating algorithms. To that end, we provide a method that adapting interpolation technique in generating a new contour from sets of control point in myocardial. The performance algorithm of tracking 
is evaluated by computing an absolute distance measure between estimated curve and ground truth curve.

\section{METHODS}

\section{A. Distance Measurement}

The first step for tracking technique evaluation is to choose sets of the parameter to be compared. In previous work of myocardial boundary tracking application, the parameters that want to be compared are the computed end systole (ES) boundary shape and the ES ground truth boundary shape. In this case, the boundary shape consists of 9 until 15 control points along myocardial boundary on both end diastoles (ED) and ES. In this tracking application, these ES control points are obtained from the computing algorithm of the ED control points. Whereas the ground truth ES control point are usually independent of the computing result and difficult to track from the initial control points in ED boundaries. In other word, the computed controls points and the ground truth control points have no correlation in tracking. Both of the control points are independent in generating process.

Let the distance between two curve $p$ and $p^{0}$ be denoted by $d\left(p, p^{0}\right)$ where $p$ is sets of computed boundaries curve and $p^{0}$ is sets of ground truth boundaries curve. Each of curves is represented by sets of control point $p=\left\{p_{1}, p_{2}, p_{3}, \ldots, p_{n}\right\}$ and $p^{0}=\left\{p^{0}{ }_{1}, p^{0}{ }_{2}, p^{0}{ }_{3}, \ldots, p_{n}{ }_{n}\right\}$, where each $p$ and $p^{0}$ is a pair of $x$ and $y$ coordinates of a control point of the curve. The distance of $p$ from the ground truth $p^{0}$ that computed using MSSD [8] is

$$
d\left(p, p^{0}\right)=\frac{1}{2 n}\left(\sum_{k=1}^{n} \min \left(x_{k}-x_{k}^{0}\right)^{2}+\sum_{k=1}^{n} \min \left(y_{k}-y_{k}^{0}\right)^{2}\right)
$$

The distance of $p$ from the ground truth $p^{0}$ that computed using MAD [4] is

$$
d\left(p, p^{0}\right)=\frac{1}{n} \sum_{k=1}^{n} \sqrt{\left(x_{k}-x_{k}^{0}\right)^{2}+\left(y_{k}-y_{k}^{0}\right)^{2}}
$$

where $p=\left\{\left(x_{1}, y_{1}\right),\left(x_{2}, y_{2}\right), \ldots,\left(x_{n}, y_{n}\right)\right\}$ is sets of computed control points and $p^{0}=\left\{\left(x_{1}^{0}, y^{0}{ }_{1}\right),\left(x^{0}{ }_{2}, y^{0}{ }_{2}\right), \ldots\right.$, $\left.\left(x^{0}{ }_{\mathrm{n}}, y^{0}{ }_{\mathrm{n}}\right)\right\}$ is sets of ground truth control points.

A proper distance measure is needed in comparing performance of the tracking algorithm. Both of evaluation algorithms are not proper with common tracking application that has independent control points of computed and ground truth. The control points on ES boundary are hardly tracked from the ED boundary manually by cardiologist, especially in noisy echocardiography image sequences that have many frames during the cardiac cycle. Computing distance using MSSD and MAD need a pair of control point that have point correspondence [4] as shown in Figure 1(a). A set of point correspondence traced form ED to ES is hardly generated in clinical data though by expert cardiologist.

In [9], no correspondence control points are used in comparing the computed and the ground truth control point. The nearest distance between control points and edge in another curve is used as the corresponding distances measure as shown in Figure 1(b). The deficiency of this technique while implementing in common myocardial tracking application is that nearest points is not eternally the proper distance to the shortest distance to the boundary. This technique is used properly to evaluate points distance in boundary detection evaluation that has contours of myocardial as in $[3,10]$.

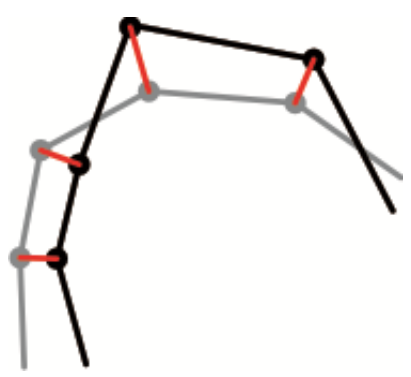

(a)

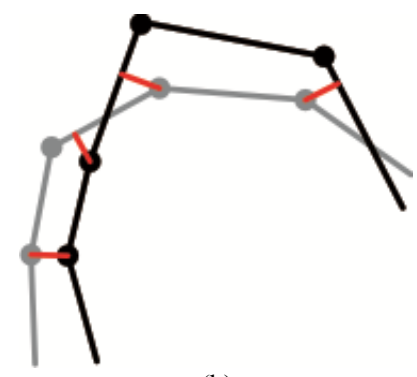

(b)
Fig. 1 Comparison of shape distance measure using (a) a pair control point distance (b) shortest distance between control point and curve

\section{B. Interpolation Curve Distance}

B-spline interpolation is used to solve the distance measurement problem in myocardial boundary tracking by generating a new boundary contour from sets of control point. In such case, boundary contour will correct the distance measure between control point and curve. The bspline interpolation will correct the error distance measure completely, but note that this correction is a smooth approach compare with the above methods. In other word, the curves distance measure is average of absolute shortest distance between control point on the first curve and interpolation point on other curve. The distance of the curve can be written as

$$
\begin{gathered}
d\left(p, p^{0}\right)=\frac{1}{2}\left(\frac{1}{n} \sum_{k=1}^{n} d\left(p_{k}, P^{0}\right)+\frac{1}{n} \sum_{k=1}^{n} d\left(p_{k}{ }^{0}, P\right)\right) \\
P=\mathrm{B}(p) \quad \text { and } P^{0}=\mathrm{B}\left(p^{0}\right)
\end{gathered}
$$

where $P$ and $P^{0}$ the new boundary contour that generated using wide de Boor's interpolation from a set of computed $p=\left\{p_{1}, p_{2}, p_{3}, \ldots, p_{n}\right\}$ and ground truth control $p^{0}=\left\{p_{1}^{0}, p^{0}{ }_{2}, p^{0}{ }_{3}, \ldots, p^{0}{ }_{n}\right\}$ respectively.

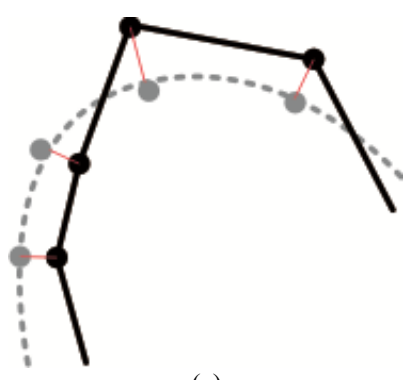

(a)

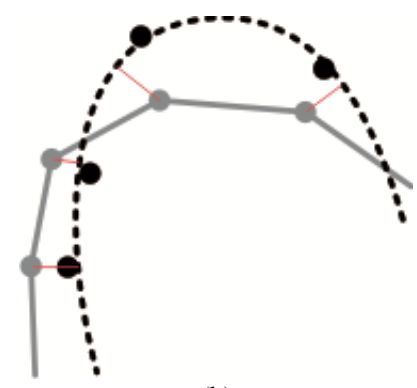

(b)
Fig. 2 Comparison of shape distance measure using shortest distance between (a) control point of computed boundary and interpolation curve of ground truth (b) control point of ground truth and interpolation of computed boundary. 
Figure 2 illustrate the curve distance measure between control point and interpolation control points from other curve that drawn as the dotted line. In this paper, 10 points are interpolated between two consecutive points. The total of control point will increase 10 times after finishing interpolation process.

The minimum Euclidian distance is calculated to compute distance between point and the nearest edge in interpolated point as follow

$$
d\left(p_{k}, P^{0}\right)=\min _{j}\left\{\sqrt{\left(x_{k}-x^{0}{ }_{j}\right)^{2}+\left(y_{k}-y_{j}^{0}\right)^{2}}\right\}
$$

where subscript $k$ and $j$ denote the index of point of the original curve and interpolation curve respectively.

\section{Data Preparation}

In this experiment, synthetic and real clinical echocardiographic was used to evaluate myocardial boundary tracking performance. Short axis view of human cardiac was used as a model of B-mode simulated echocardiographic image as shown in fig 3(a). Using the Field II ultrasound simulation software [11, 12], the simulated echocardiographic image is generated which a linear scan was set at $3.0 \mathrm{MHz}$ with a 128-element transducer, using 64 element phase arrays with Hanning apodization and the frequency of sampling at $100 \mathrm{MHz}$. This software comprises several algorithms to create ultrasound simulated images. It has the ability to generate a realistic ultrasound transducer that transmits and receives ultrasound signal through biological tissue.

The synthetic image sequence consist of 8 frames that obtained by implementing motion vector as shown in fig 3(b) to initial synthetic image. In the synthetic image model, the heart's left ventricle chamber is made as in the process of cardiac expanding, which the area of the cavity is getting bigger. The maximum displacement of the farthest position from the center of the cavity was set to 2.5 pixels.
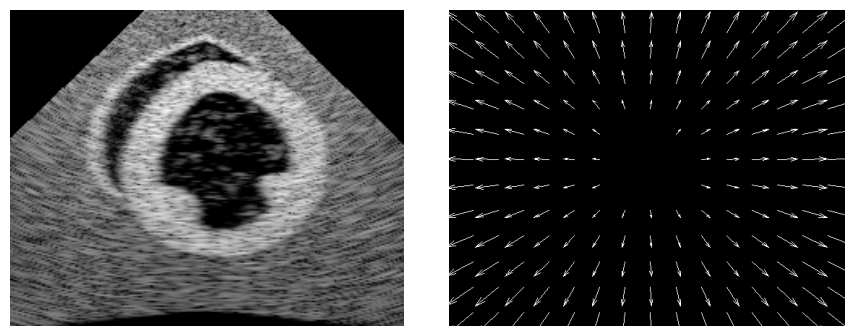

Fig. 3 (a) The initial ultrasound image was generated using the Field II ultrasound simulation software (b) velocity vector model with maximum displacement is 2.5 pixels

The clinical echocardiographic image that used to evaluate myocardial tracking performance is a sequence of digitized B-mode ultrasound image. These data were acquired from 10 healthy volunteers for each cardiac view such as parasternal short axis, parasternal long axis, 2 chambers and 4 chambers. The Acuson Squoia C512 Ultrasound Machine at Cardiac Care Unit UKM Medical Centre was used to record scan sequence. All echocardiographic images have a width and height of $384 \times 287$ pixels and same frame rate.
In this paper, myocardial boundary tracking is implemented by computing control point displacement in terms of angle and magnitude of points in motion vector space. A set of control points was chosen along the myocardial boundary on the first frame of echocardiographic sequence. The total displacement of all frames is obtained by adding the displacement of consecutive frames and the initial position of points in the end diastole cycle.

Let $\boldsymbol{B}(x, y, t)$ be any position of the myocardial boundary at time $t$ and position $(x, y)$ in 2D echocardiographic image. Time $t$ starts at 0 in ED cycle and stops at $T$ in ES of single cardiac cycle. The position of the myocardial boundary at time $t$ and position $(x, y)$ can be written as follows [7]

$$
\boldsymbol{B}(x, y, t)=\boldsymbol{B}_{\boldsymbol{o}}(x, y)+\Delta \sum_{i=0}^{t} \boldsymbol{U}_{\boldsymbol{i}}(u, v)
$$

where $\Delta$ is the time interval between consecutive frames, $\boldsymbol{B}_{\mathbf{0}}$ is the position of the myocardial boundary in the end diastole of the cardiac cycle $(\mathrm{t}=0)$, and $\boldsymbol{U}_{\boldsymbol{i}}$ is the motion vector space that computed using optical flow. In this paper, eight optical flow techniques are used to generate motion vector and compared their performance in myocardial boundary tracking.

\section{RESULT AND DISCUSSION}

In this paper, the proposed performance evaluation algorithm is tested to quantify myocardial boundary tracking technique. Performance of myocardial boundary tracking is computed as curve distance measure between estimated and ground truth control point. Smaller curve distance means that the computed control points are close to ground truth. It means that the myocardial tracking algorithm is more accurate in computing the subsequent myocardial position. In this experiment, we used the synthetic echocardiographic image sequence and real echocardiographic clinical data for comparing the performance.

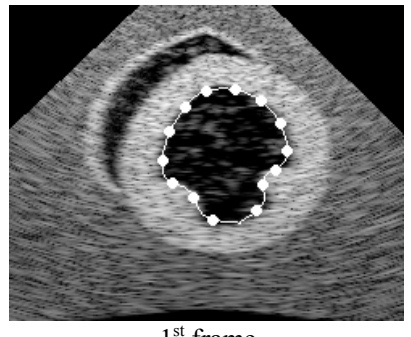

$1^{\text {st }}$ frame

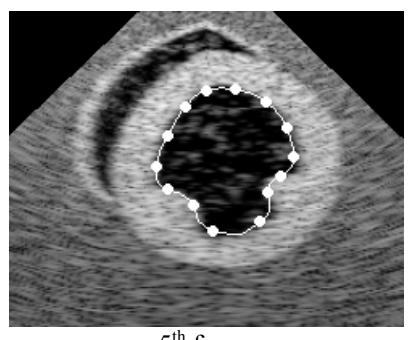

$5^{\text {th }}$ frame

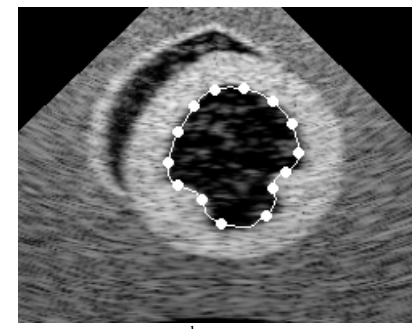

$3^{\text {rd }}$ frame

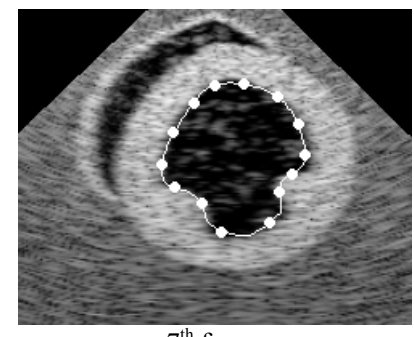

$7^{\text {th }}$ frame
Fig. 4 The ground truth of myocardial boundary tracking for $1^{\text {st }}, 3^{\text {rd }}, 5^{\text {th }}$ and $7^{\text {th }}$ frame on synthetic ultrasound with parasternal short axis view

Figure 4 shows the ground truth of myocardial boundary tracking that used for evaluating the tracking algorithm. 
Fourteen control points is used which the movement of them is known exactly in magnitude and angle. The $x-y$ coordinate of the control point of each frame is estimated by adding the $x-y$ coordinate of previous control point and the motion vector. In this experiment, the same motion vector as shown in Figure 3(b) is used for each frame transition so the control point will move with constants magnitude and direction during the cycle.

Figure 5 is shown an example of myocardial boundary tracking result in real echocardiography image sequence. The boundary tracking shows the compression process in the left ventricle of human normal cardiac. The boundary tracking result follows the area of the cavity which becomes smaller along the compression process.

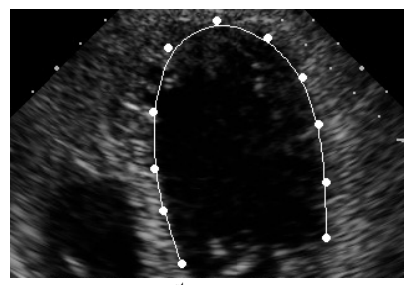

$1^{\text {st }}$ frame

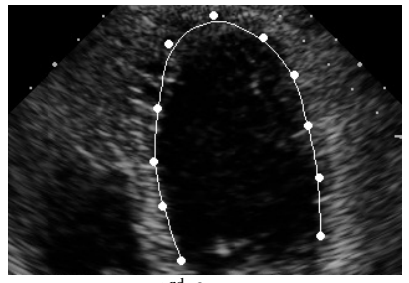

$3^{\text {rd }}$ frame

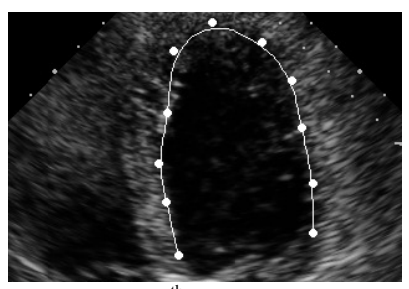

$5^{\text {th }}$ frame

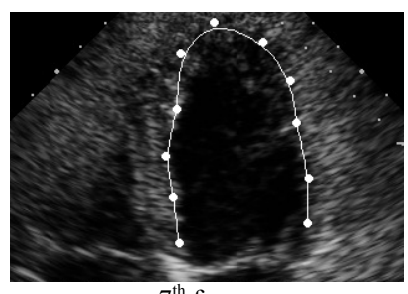

$7^{\text {th }}$ frame

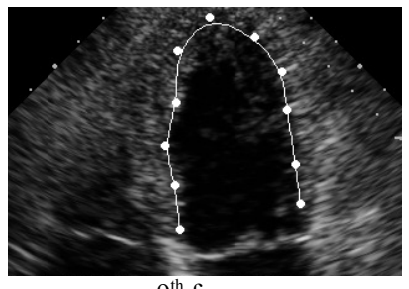

$9^{\text {th }}$ frame

(a)

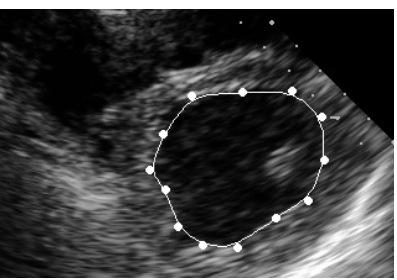

$1^{\text {st }}$ frame

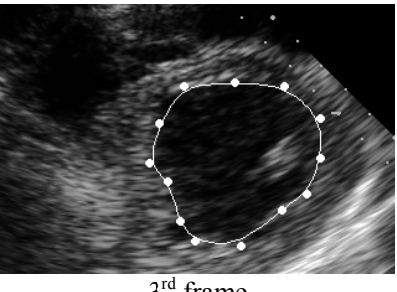

$3^{\text {rd }}$ frame

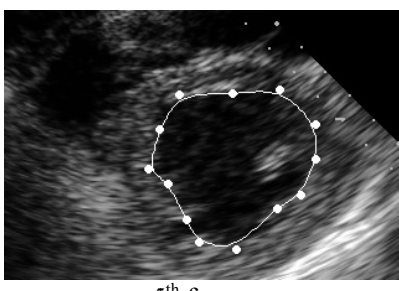

$5^{\text {th }}$ frame
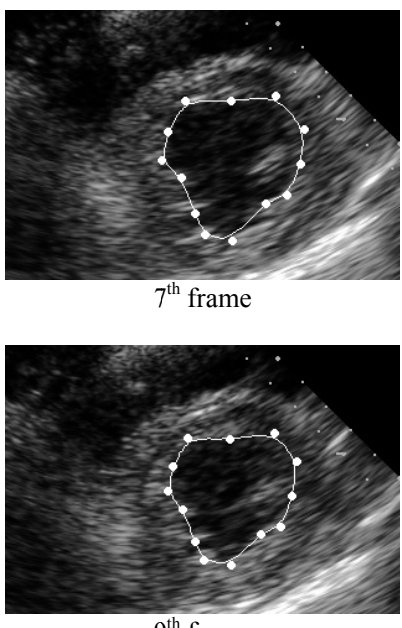

$9^{\text {th }}$ frame

(b)
Fig. 5 Example of Myocardial boundary tracking result for $1^{\text {st }}, 3^{\text {rd }}, 5^{\text {th }}, 7^{\text {th }}$ and $9^{\text {th }}$ frame on (a) 3 chamber cardiac view and (b) parasternal short axis view
Figure 5 shows the comparison of curve distance measure result on synthetic (left bar) and real clinical (right bar) of echocardiographic sequence using eight optical flow methods. The graphic subscript of HS, LK, LKPyr, TG, GF, Brox and BA denote for Horn Shuck [13], Lucas Kanade [14], Pyramidal Lucas Kanade [15], Gautama [16], Gurner Farneback [17], Brox [18] and Black Anandan [19] respectively. The curve distance measure is represented by an error value between estimated control point using equation 6 and ground truth control point. In this first experiment, all methods could track the myocardial boundary with error below 0.5 pixel except the Brox [18] method. The smallest error is provided by Gurner Farneback and proposed method. The boundary tracking result on synthetic data is very accurate given by both methods. Both of methods have an average error value 0.076 pixel of all control points along the myocardial boundary.

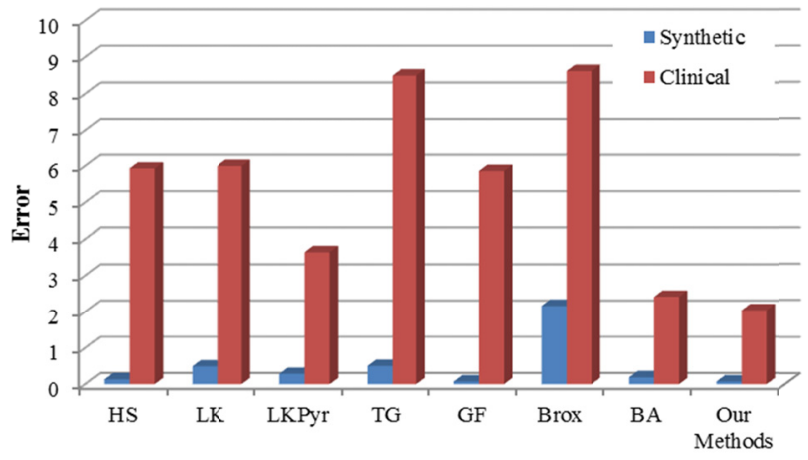

Fig. 6 Performance evaluation comparison of 8 optical flow techniques in tracking myocardial boundary on synthetic and real echocardiographic image sequence

Lastly, ten echocardiographic image sequences are used to compare the performance of the tracking algorithm in real clinical data. This data consist of 4 view of human cardiac variations such as parasternal long axis, short axis, 4chamber and 2-chamber view. Each cardiac view has 9 - 14 control points of myocardial curve. The image sequence consist ten frames for each cardiac cycle (ED to ES). The result of error shown in Figure 6 (right bar) is mean of error value for all cardiac view. In this experiment, our methods provide the best performance with the smallest error value. The tracking result of this method is shown in figure 5. The estimated myocardial boundary is estimated almost perfectly close to the real boundary. The proposed myocardial boundary tracking included optical flow technique was appropriate for accurate myocardial boundary tracking.

Based on the both experiment result using synthetic and real echocardiography, the performance of myocardial boundary tracking could be compared quantitatively. The performance result using synthetic data should be more reliable in case of ground truth generation whereas the real clinical data should be more reliable in case of real noisy image. In short, the curve distance measure described in here is useful and provides means of evaluating performance of myocardial boundary tracking quantitatively. 


\section{CONCLUSION}

A methodology of myocardial boundary evaluation has been developed using curve distance measure. To test the usefulness of the proposed methodology, eight motion estimation techniques were put to test and their performances are evaluated quantitatively in term of the curve distance error on sets of image sequences. Set of image database consist of synthetic and real clinical echocardiography image sequence. The result of tracking performance is an error value that representing a curve distance measure between the estimated and ground truth control point. We concluded that the curve distance measure provides means of evaluating performance of myocardial boundary tracking quantitatively.

\section{ACKNOWLEDGMENT}

The authors would like to acknowledge Universiti Kebangsaan Malaysia (Project code UKM-GUP-TKP-08-24080) for the financial support awarded for this research.

\section{REFERENCES}

[1] J. L. Barron, et al., "Performance of Optical Flow Techniques," International Journal of Computer Vision, vol. 12, pp. 43-77, 1994

[2] B. McCane, et al., "On Benchmarking Optical Flow," Computer Vision and Image Understanding, vol. 84, pp. 126-143, 2001.

[3] C. Jierong, et al., "Watershed-presegmented snake for boundary detection and tracking of left ventricle in echocardiographic images," Information Technology in Biomedicine, IEEE Transactions on, vol. 10, pp. 414-416, 2006.

[4] D. Comaniciu, et al., "Robust real-time myocardial border tracking for echocardiography: an information fusion approach," Medical Imaging, IEEE Transactions on, vol. 23, pp. 849-860, 2004.

[5] I. Mikic, et al., "Segmentation and tracking in echocardiographic sequences: active contours guided by optical flow estimates," Medical Imaging, IEEE Transactions on, vol. 17, pp. 274-284, 1998.
[6] K. Althoff, et al., "Tracking contrast in echocardiography by a combined snake and optical flow technique," in Computers in Cardiology 2000, 2000, pp. 29-32.

[7] A. H. Saputro, et al., "Enhancement of Myocardial Boundary Tracking Using Wavelet-based Motion Estimation," Journal of Information and Computational Science vol. 8, pp. 1779-1792, 2011.

[8] Y. S. Akgul, et al., "Automatic extraction and tracking of the tongue contours," Medical Imaging, IEEE Transactions on, vol. 18, pp. 1035-1045, 1999.

[9] Y. S. Akgul and C. Kambhamettu, "A coarse-to-fine deformable contour optimization framework," Pattern Analysis and Machine Intelligence, IEEE Transactions on, vol. 25, pp. 174-186, 2003.

[10] V. Chalana, et al., "A multiple active contour model for cardiac boundary detection on echocardiographic sequences," Medical Imaging, IEEE Transactions on, vol. 15, pp. 290-298, 1996.

[11] J. A. Jensen, "Field: A Program for Simulating Ultrasound Systems," Medical \& Biological Engineering \& Computing, vol. 34, pp. 351353, 1996.

[12] J. A. Jensen, Ultrasound imaging and its modeling. Verlag: Springer, 2002.

[13] B. K. P. Horn and B. G. Schunck, "Determining optical flow," Artificial Intelligence, vol. 17, pp. 185-203, 1981.

[14] B. D. Lucas and T. Kanade, "An iterative image registration technique with an application to stereo vision," International Joint Conference on Artificial Intelligence (IJCAI), pp. 674 - 679, 1981.

[15] J.-Y. Bouguet. (2000, Pyramidal Implementation of the Lucas Kanade Feature Tracker

[16] T. Gautama and M. A. Van Hulle, "A phase-based approach to the estimation of the optical flow field using spatial filtering," IEEE Transactions on Neural Networks, vol. 13, pp. 1127-1136, 2002.

[17] G. Farneback, "Very high accuracy velocity estimation using orientation tensors, parametric motion, and simultaneous segmentation of the motion field," in Computer Vision, 2001. ICCV 2001. Proceedings. Eighth IEEE International Conference on, 2001, pp. 171-177 vol.1.

[18] T. Brox, et al., "High Accuracy Optical Flow Estimation Based on a Theory for Warping," in 8th European Conference on Computer Vision, Prague, Czech Republic, 2004, pp. 25-36.

[19] M. J. Black and P. Anandan, "A framework for the robust estimation of optical flow," in Computer Vision, 1993. Proceedings., Fourth International Conference on, 1993, pp. 231-236. 